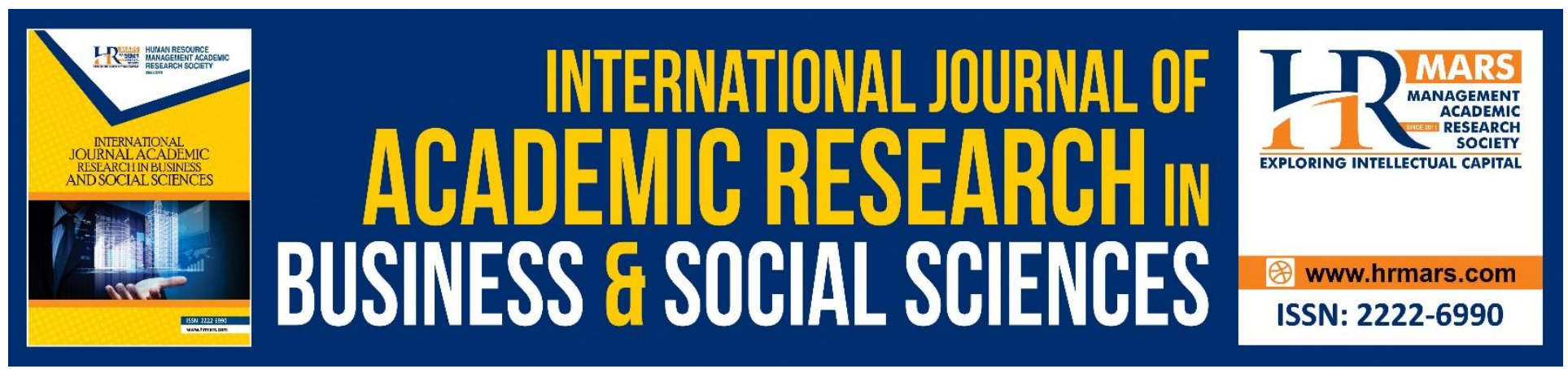

\title{
Software Define Network Applications on Top of Blockchain Technology
}

\author{
Muhammad Johar Jaafar
}

To Link this Article: http://dx.doi.org/10.6007/IJARBSS/v8-i6/4312

DOI: $\quad 10.6007 /$ IJARBSS/v8-i6/4312

Received: 24 May 2018, Revised: 19 June 2018, Accepted: 29 June 2018

Published Online: 08 July 2018

In-Text Citation: (Jaafar, 2018)

To Cite this Article: Jaafar, M. J. (2018). Software Define Network Applications on Top of Blockchain Technology. International Journal of Academic Research in Business and Social Sciences, 8(6), 1188-1194.

Copyright: (C) 2018 The Author(s)

Published by Human Resource Management Academic Research Society (www.hrmars.com)

This article is published under the Creative Commons Attribution (CC BY 4.0) license. Anyone may reproduce, distribute, translate and create derivative works of this article (for both commercial and non-commercial purposes), subject to full attribution to the original publication and authors. The full terms of this license may be seen at: http://creativecommons.org/licences/by/4.0/legalcode

Vol. 8, No. 6, June 2018, Pg. 1188 - 1194

http://hrmars.com/index.php/pages/detail/IJARBSS

JOURNAL HOMEPAGE

Full Terms \& Conditions of access and use can be found at http://hrmars.com/index.php/pages/detail/publication-ethics 


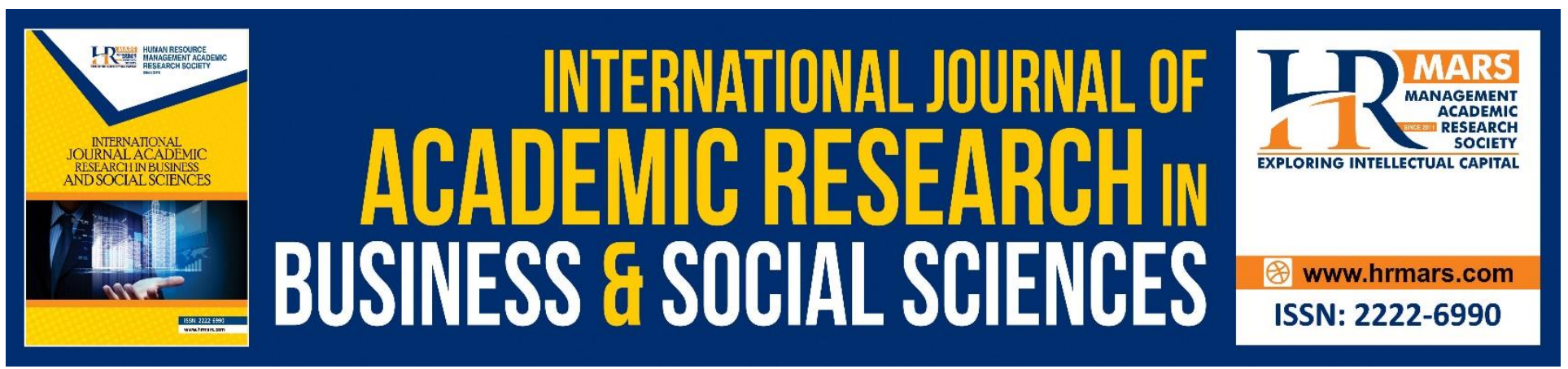

\title{
Software Define Network applications on top of Blockchain technology
}

\author{
Muhammad Johar Jaafar \\ Universiti Pertahanan Nasional Malaysia, Malaysia
}

\begin{abstract}
Nowadays technologies are rapidly growth and it cover many areas. The traditional or legacy network architecture is no longer suited to meet today requirement ever since cloud era appeared. In order to fulfill the gap, the paradigm has shifted to edge computing where the usage of micro-services become mainstream. The needs of using edge computing become crucial when intruder, and malicious become the sentinel of all disease in network infrastructure. Thus, with the usage of blockchain in SDN infrastructure technology it could be the game changer. Whereby it has the mechanism for long term basis, long term integrity, auditability, lineage, Secure provenance and quantum immunity. All the attribute component can be a single platform where it trustful and transparent. Without doubt, it also improves the environment for controlling the threat. In this case, it could be the best platform for financial services industry to adapt. The other significant benefits can be shared in other domain such as in loT infrastructure, and edge computing area. Therefore, it can outweigh the additional compliances costs involved in establishing the ecosystem (EY Assurance, 2016). In laymen terms, the future of network can be handle at front end (surface layer) instead of distributing the task over others. Thus, it improves a new business opportunity on each applied solution.
\end{abstract}

Keywords: Software Define Network, Blockchain, Switch, Network Security, Edge Computing.

\section{Introduction}

Nowadays technologies are rapidly growth and it cover many areas. The traditional or legacy network architecture is no longer suited to meet today requirement ever since cloud era appeared. In order to fulfill the gap, the paradigm has shifted to edge computing where the usage of micro-services become mainstream. Therefore, the Software Define Networking become prominent due to the transformation of the network architecture and underlying processes. Software Define Network or SDN has flexible mechanism, whereby it can gain unprecedented network control, programmability and automation for it to work under heavy or low concurrent resources. In SDN, the protocol involve is OpenFlow. The OpenFlow protocol work as medium agent in interoperability and also protocol that use together with Controller (Eg. Trema, Ryu, POX, NOX, Floodlight, Opendaylight and etc). When it come to blockchain, the capability is extent to the nature of blockchain itself. Blockchain or permanent ledger, create strong encrypted properties, that no entity can leverage or tempered the 
INTERNATIONAL JOURNAL OF ACADEMIC RESEARCH IN BUSINESS AND SOCIAL SCIENCES Vol. 8, No. 6, June 2018, E-ISSN: 2222-6990 @ 2018 HRMARS

detail. Thus, to penetrate the chain is nearly impossible to do. Aside front that, the mechanism such as fast reconciliation is beneficial towards applications and users. Since the usage of SDN is now at Data center level, the Cloud Computing technology such as Openstack should start using blockchain as well. With this, it can assure various industries and, far reaching implications for socioeconomic. In this paper, the researcher also wants to share on possible method approach and implementation blockchain technology in SDN environment collaborating cloud computing technology at data center level. Aside from this, the researcher provide a detailed description of blockchain, SDN, and technologies involved.

\section{Background}

In this paper, the main elements that involved are SDN, Blockchain and Openstack. However, the application are varies and the first researcher decides on using python, json and javascript as the main language. Thus, from this view, the application that available for such criteria are SDN, Learning Switch, Blockchain and its possible approach.

\section{A. Software Define Network}

Software Define Network or SDN is a new paradigm in nowadays networking. It allows network administrators to manage network services through abstraction of higher-level functionality. The SDN works by proprietary on top of software commodity. Thus, the decoupling or decentralization mechanism is able to pro the system that makes decisions about where traffic is sent (the control plane) from the underlying systems that forward traffic to the selected in next destination (the data plane). In other words, SDN is an architecture purporting to be dynamic, manageable, cost-effective, and adaptable, seeking to be suitable for the high-bandwidth, dynamic nature' of today's applications. SDN architectures decentralized the network control and forwarding functions, thus it, become directly programmable for underlying infrastructure from applications and network services.

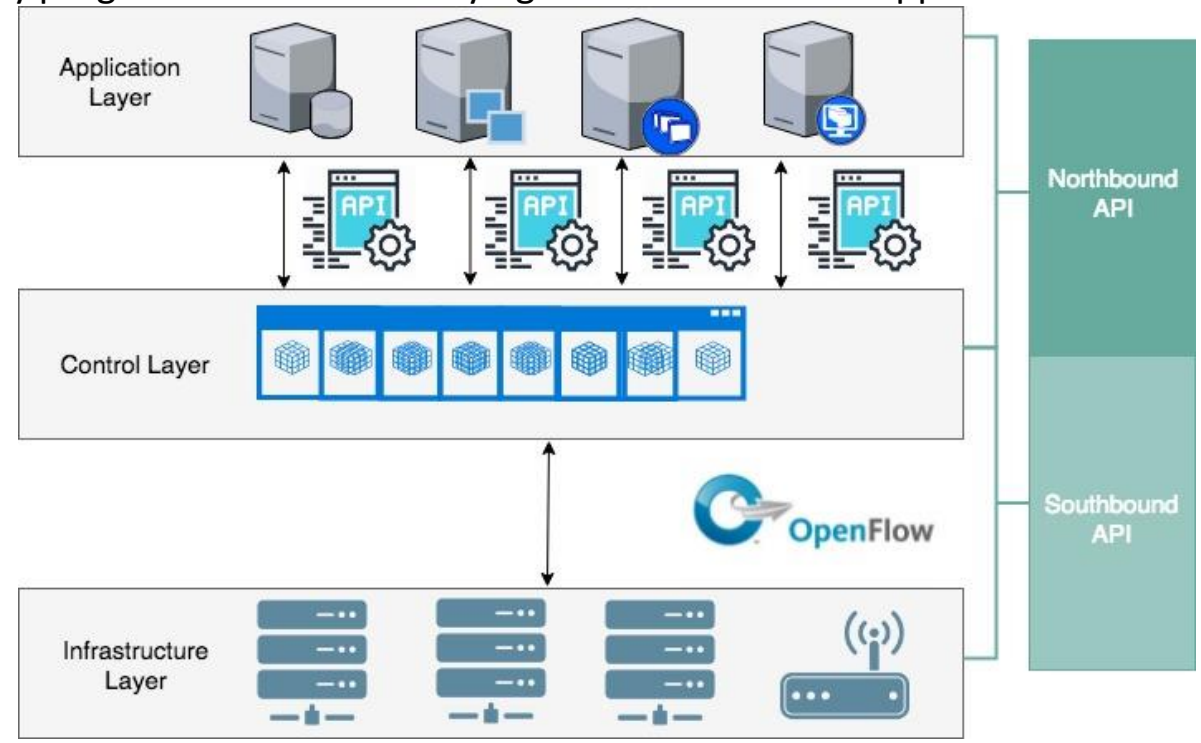

Figure 1. Diagram of software Define Architecture.

As stated in figure 1, the decentralization or decouple happen in between the two zone, Northbound and Southbound zone. This zone handle the API in between them. From the top, the SDN Controller communicate (towards control layer) to segregate the anomalies and allow only SDN API connection 
INTERNATIONAL JOURNAL OF ACADEMIC RESEARCH IN BUSINESS AND SOCIAL SCIENCES Vol. 8, No. 6, June 2018, E-ISSN: 2222-6990 @ 2018 HRMARS

to communicate with Control layer. This is where all the network application and neutron (Openstack application) communicate, as well all external application like DDOS protection or Network Application Orchestration and Services is placed (such as chef, and puppet). In Control Layer, all the received API will map, stats track and other network function capability to process and distribute. The service abstraction layer ( $\mathrm{SAL}$ ), start to invoke (conversion) all attribute into singular form to make the southbound recognize this function in respective element (according the functions). In infrastructure layer, the receive element accept according to the service and directly connected to device. This connection is supervised or provision by OpenFlow protocol. Thus, it must be connect before running the services.

The OpenFlow creates separate policies that universally program the entire network in single fabric. The capability extend the provision into underlying data plane to device-forwarding table. The other function that can be found is the capability of isolation whereby it provide the regular production traffic and traffic untouched. Second function is virtualized and programmable, which different flows has different way to process. By having this, it can reduce the wiring in the Network closet and reduce the cost (Standford University, 2010).

According to various of SDN framework case study, few controller are used but only few are really compatible with blockchain mechanism. The controller involved are OpenDaylight, RYU, ONOS, vneio and Faucet.

However, the appeal mechanism haven't reach to its peak for production usage with blockchain. It is because it involve with various stack services and it work simultaneously (Tech Target, 2015).

\section{B. Learning Switch}

Learning switch or Ethernet switch, known to link among other network devices such as router or among switches. This hardware component has been use for many type of network both small or larger networks and it use commonly in data center. Most of the time, the learning processes are involving learning process and transfer. In this research, the term usage is really refer to learning switch which mainly use for learning and transfer (however it can evolve into router, routing switch and network slices) due to the mechanism of learning switch that takes effect widely applied.

When look back at traditional learning switch (in Figure 2), the learning process works by host send a frame to another devices through the switch. The switch captures the source MAC address of the frame and the interface that received. When it confirms the MAC addresses it will immediately lookup the entries in lookup table. By default, it set an address entry 300 seconds ( 5 minutes). The timer will get rid unused entries in order to keep lookup process short and fast (Paul, 2013).

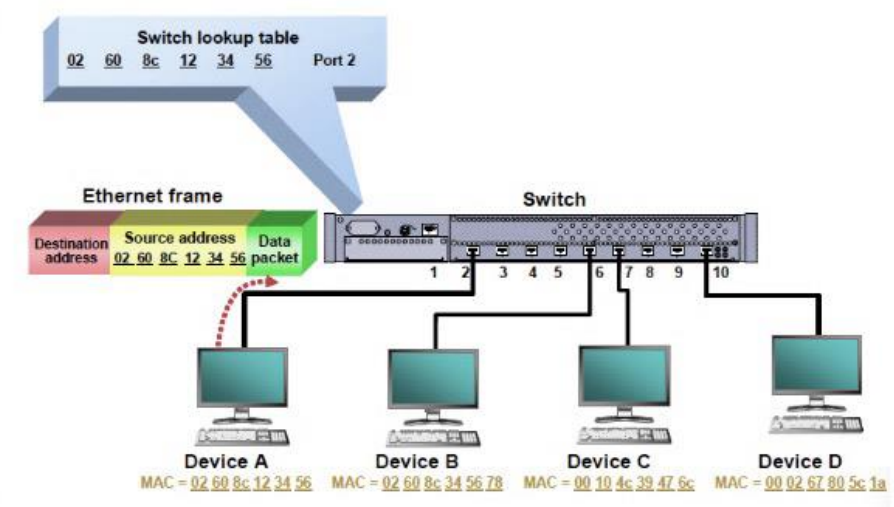


Figure 2: Legacy Ethernet Switches Work (Paul, 2013).

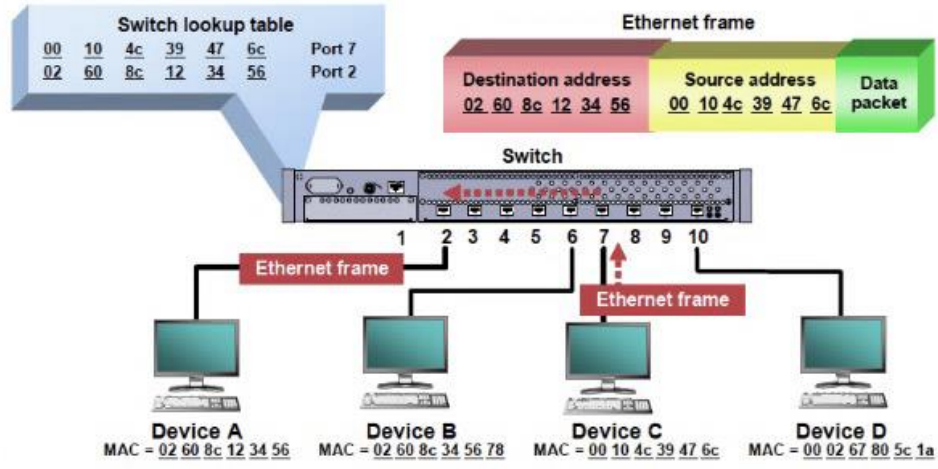

Figure 3: Learning, Forwarding and filtering processes in legacy switch

The switches later learn by flooding the single frame out all in order to learn the MAC address destination switch (by using Address Resolution Protocol (ARP) request). By flooding the frame it can filter and forward at the same time in the switch lookup table. When the switch recognizes the device MAC address it will reply in towards the respective port. For the example in Figure 3, it only occurs that it will filter only target MAC Address for this cases from port 2 to port 7. Other than port 7 will be filtered out and it will drop in the lookup table. By doing this, no one else can look at the contents of the frame. However, it's a little different in OpenFlow, whereby any of switches involving will become learning switch (when apply varies of function inside the module can become router, routing switch and network slice). The learning method is same, which, it includes the packet from host, to learn the position of the host on the network and, transfer by using in positioning information that has been learned via flood and forward packet to the destination host. However, in OpenFlow it involve two different packet to be send and the flood mechanism are bit different which the flow mod or flood manager will be flood simultaneously to all exist port at the first place and waiting for the packet to insert to the Database or Forwarding Database (FDB). Whenever the packet enters to the controller via OpenFlow connection. The OpenFlow packet will declare packet-in because of the flow table still empty, from that moment the packet will join the flood pool in the controller until the controller finish checking the source and destination path. With this, the packet will register in FDB and instruct the switch to spread the PacketOut message by specifying the output port to the flooding. As a result, the packets arrive at the host.

\section{Blockchain}

Blockchain is a set of protocol that use encryption for securely storing data on a distributed network. Each block carries a list of distributed data as well a hash to the previous block. Each of it, has its own references and make an interconnection between blocks. Once it fill, it push to the next chunk to overflow current block to create a new block. Figure 2, is the example of creation of block and how it is interconnected and carry list of data, and hash the previous block. 
INTERNATIONAL JOURNAL OF ACADEMIC RESEARCH IN BUSINESS AND SOCIAL SCIENCES

Vol. 8, No. 6, June 2018, E-ISSN: 2222-6990 ๔ 2018 HRMARS

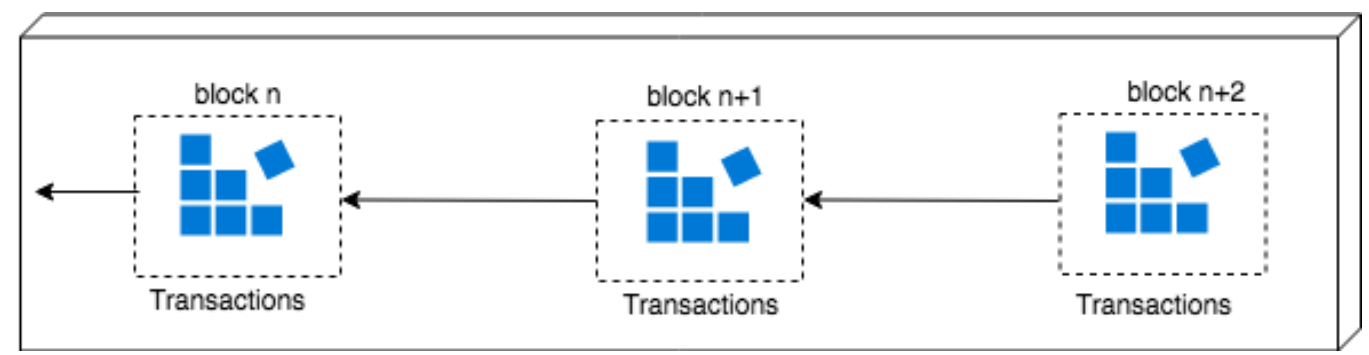

Figure 4: Transaction block and hash to previous block.

By referring the above photo in Figure 4, the blockchain mechanism work as point-to-point as standard form of framework which it goes through block by block (in general form). In executions, in blockchain bottom line, it work 3 ways. Either as public, private or consortium/federated.

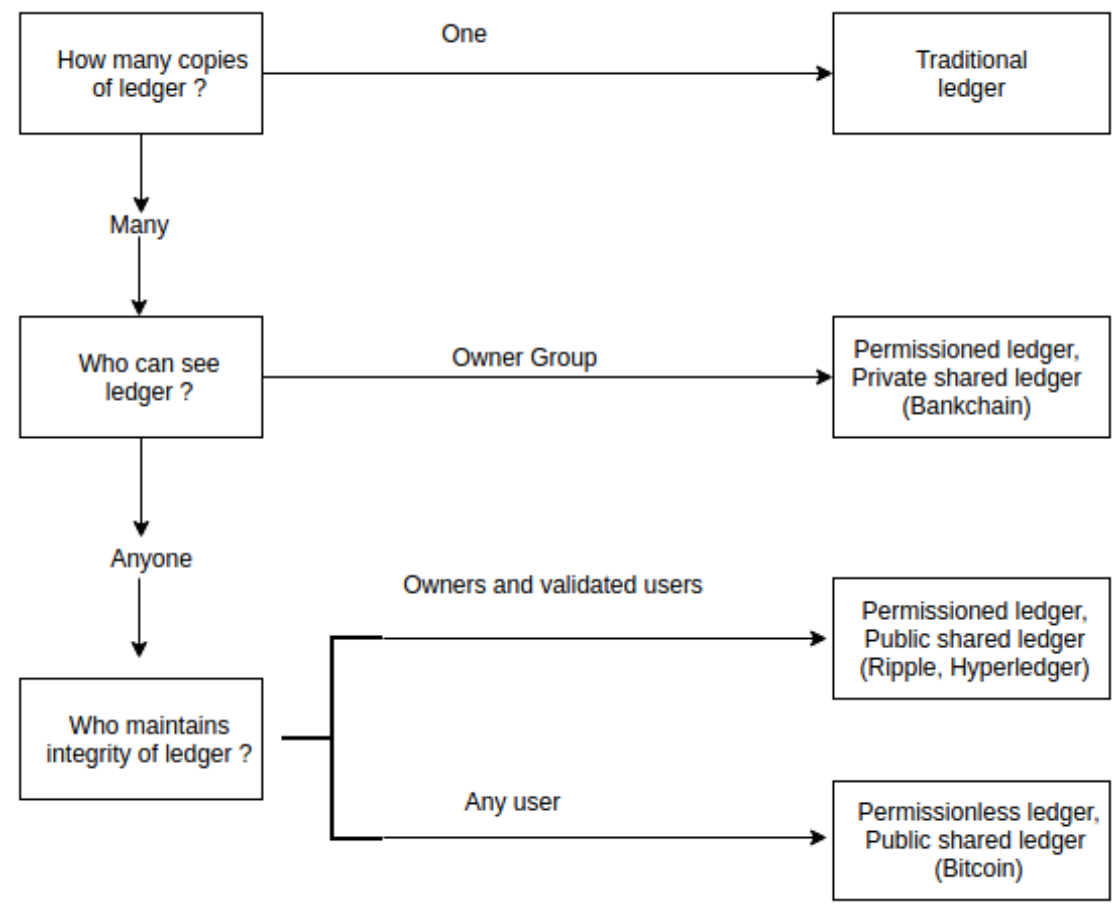

Figure 5: Flow chart of blockchain work

In figure 5, the figure shows the flow of two separate type of blockchain (in conditional form). In general, Public provide the decentralized platform that anyone can read and send, but private provide centralized under one control (Shermin Voshmgir, 2017). The consensus define the blockchain shape as permission-less blockchain or permissioned blockchain. This two shapers create the new fabric to meet the network requirement, either it can run publically or privately. In this research, the possible component to implement the block on top on of it is smart contract. The smart contract works by using hashing algorithm functions whereby it only work when both end has the same key or condition are met. The rational reason for using the smart contract because the SDN is known for its vulnerabilities over vector. Whereby, the malicious can creates entries in the network flow and change the ownerships of the network (Guardtime). Once, its infected, the controller, network element or host connected will creates a room for its penetration. However, with smart contract it can keyed policy that only registered can provisioning the entire infrastructure. 
INTERNATIONAL JOURNAL OF ACADEMIC RESEARCH IN BUSINESS AND SOCIAL SCIENCES

Vol. 8, No. 6, June 2018, E-ISSN: 2222-6990 @ 2018 HRMARS

\section{Conclusion}

In edge of computing, there is no single solution that able to mitigate such intrusion. However, with the usage of blockchain in SDN infrastructure technology it could be the game changer. Whereby it has the mechanism for long term basis, long term integrity, auditability, lineage, Secure provenance and quantum immunity. All the attribute component can be a single platform where it trustful and transparent. Without doubt, it also improve the environment for controlling the threat. In this case, it could be the best platform for financial services industry to adapt. The other significant benefits can be shared in other domain such as in loT infrastructure, and edge computing area. Therefore, it can outweigh the additional compliances costs involved in establishing the ecosystem (EY Assurance, 2016). In laymen terms, the future of network can be handle at front end (surface layer) instead of distributing the task over others. Thus, it improves a new business opportunity on each applied solutions.

\section{Acknowledgement}

This research work is supported by NRGS grant from Ministry of Higher Education of Malaysia.

\section{Corresponding Author}

Omar Zakaria

Faculty of Defence Science and Technology

National Defense University of Malaysia (UPNM)

Sg Besi, Kuala Lumpur, 57000, Malaysia

Email:omar@upnm.edu.my

\section{References}

Standford University. (2010, January 5). Overview. Retrieved from Overview-CS244wiki: http://yuba.stanford.edu/cs244wiki/index.php/Overview

Tech Target. (2015, April). Network's Next Sea Change, The SDN WAN. Retrieved from Tech Target: https://searchsdn.techtarget.com/ehandbook/Is-your-network-ready-for-the-SDN-WAN

EY Assurance. (2016). EY - Implementing blockchains and distributed infrastructure. Retrieved from EY Assurance: http://www.ey.com/Publication/vwLUAssets/EY-implementing-blockchainsand-distributed-infrastructure/\$FILE/EY-implementing-blockchains-and-distributedinfrastructure.pdf

Shermin Voshmgir, V. K. (2017). blockchain: A beginner Guide. blockchainhub.net.

Paul, S. (2013). How do switches work. Retrieved from Global Knowledge: http://www.globalknowledge.com.eg/about-us/Knowledge- Center/Article/How-doSwitches-Work/

Guardtime. (n.d.). Use of a globally distributed blockchain to secure SDN. Retrieved from Guardtime: https://www.weusecoins.com/assets/pdf/library/Guardtime\%20\%20Global\%20Industrial\%20Blockchain.pdf 